\title{
Modelos de enseñanza de los algoritmos de la división de fracciones
}

\author{
Bernardo Gómez Alfonso, Universidad de Valencia (España) \\ Olimpia Figueras Mourut de Montppellier, Centro de Investigación y de Estudios \\ Avanzados del IPN (México) \\ Mauricio Contreras del Rincón (post mortem auctoris), Universidad de Valencia \\ (España)
}

Recibido el 5 de enero de 2016; aceptado el 16 de enero de 2016.

\section{Modelos de enseñanza de los algoritmos de la división de fracciones}

\section{Resumen}

La identificación de aspectos especificos considerados parte de los elementos constitutivos de los modelos de enseñanza de los algoritmos para la división de fracciones es uno de los objetivos principales del estudio cuyos resultados se exponen en este artículo. Con la intención de rastrear esos elementos a lo largo del tiempo se han analizado textos escritos para la enseñanza de las matemáticas de los siglos XIII a XXI. El escrutinio de estos documentos se sustenta en análisis históricoepistemológico y de contenido. La información obtenida de este tipo de indagaciones, además de contribuir a la historia de la educación matemática conforma un corpus valioso para la formación de profesores y los diseñadores de currículo.

Palabras clave. fracciones; división; historia de la educación matemática; análisis de textos; análisis de contenido

\section{Modelos de ensino dos algoritmos de divisão de frações}

\section{Resumo}

A identificação dos aspectos especificos considerados parte dos elementos constitutivos de modelos de ensino da divisão de frações é um dos principais objetivos do estudo, cujos resultados são apresentados neste artigo. Com a intenção de rastrear esses elementos ao longo do tempo foram analisados textos para o ensino de matemática no décimo terceiro a vinte. A análise destes documentos é baseado no análise histórica-epistemológica e conteúdo. As informações obtidas a partir de tais investigações, além de contribuir para a história de educação matemática forma um corpus valioso para a formação de professores e designers de currículo.

Palavras chave. frações; divisão; história da educação matemática; análise de texto; análise de conteúdo

\section{Teaching models for algorithms of fraction's division}

Para citar: Gómez, B., Figueras, O., Contreras, M. (2016). Modelos de enseñanza de los algoritmos de la división de fracciones. Avances de Investigación en Educación Matemática, 9, 43 - 63.

(C) Sociedad Española de Investigación en Educación Matemática (SEIEM). www.seiem.es 


\begin{abstract}
The identification of constituent elements of the algorithms for fraction division's teaching models is one of the main objectives of the study whose results are reported in this article. Texts for teaching mathematics written during the 13th to 21 st centuries were analysed in order to track the aforementioned components over time. Documents' scrutiny was based on historical-epistemological analysis, as well as on content analysis. Information obtained from such inquiries, contributes to the history of mathematics education and forms a valuable corpus for teacher training and curriculum designers.
\end{abstract}

Key words. fractions; division; history of mathematics education; text analysis; content analysis

\title{
Modèles de enseignement d'algorithmes de division de fractions
}

\section{Résumé}

L'identification des aspects spécifiques considérés comme faisant partie des éléments constitutifs de modèles d'enseignement de la division de fractions es l'un des principaux objectifs de l'étude dont les résultats son rapportés dans cette article. Avec l'intention de suivre ces éléments au fil du temps ont été analysés textes pour l'enseignement des mathématiques dans la treizième á vingt. L'examen de ces documents est basée sur analyse historique-épistémologique et de contenu. L'information obtenue à partir de telles études, en plus de contribuer à l'histoire de l'enseignement de mathématiques forme un corpus précieux pour la formation des enseignants et les concepteurs de programmes.

Paroles clés. fractions; division; histoire de l'enseignement des mathématiques; analyse de textes; analyse de contenu

\section{Introducción}

Enmarcado en los proyectos de investigación que se desarrollan en el grupo de Pensamiento Numérico y Algebraico (PNA) de la Universidad de Valencia se encuentra el estudio de los fenómenos de enseñanza y aprendizaje de los conceptos aritméticos y algebraicos que son comunes a los currícula de la enseñanza primaria y secundaria. En este ámbito Mauricio Contreras hizo pesquisas que versan sobre distintos aspectos relacionados con la división de fracciones que lo condujeron a la obtención del grado de Doctor en esa institución (Contreras, 2012).

Los autores de este artículo compartieron horas y horas de reflexión y discusión con Mauricio y pretenden exponer en este documento una parte del trabajo que realizó, en concreto aquel que plasmó en un apartado de su tesis sobre los algoritmos de la división de fracciones, y al mismo tiempo ampliar sus resultados dándole de ese modo continuidad a las indagaciones que él hizo.

La pertinencia del estudio que se describe en este artículo reside en la intención práctica de aportar información fundamentada para uso de los profesores y de los diseñadores del currículo, y en este caso particular, la de dar cuenta de modelos de enseñanza sobre los algoritmos de la división de fracciones que se hayan sometido a la prueba de la investigación didáctica.

\section{Marcos de referencia teórica, metodológica y empírica}

Uno de los acercamientos teóricos y metodológicos utilizado en las líneas de investigación desarrolladas en el PNA de la Universidad de Valencia es la elaboración recursiva de Modelos Teóricos Locales (MTL) (Filloy, Puig y Rojano, 2008). El esquema general de un estudio en el cual se pretende construir un MTL se desglosa en dos fases que corresponden, una a la descripción de modelos de enseñanza existentes 
en el sistema escolar, y la otra a la experimentación para la indagación sobre las relaciones entre modelos de enseñanza existentes y las actuaciones de los alumnos.

La investigación que se describe en este artículo corresponde a la primera fase de la construcción de un MTL, principalmente interesa el componente de los Modelos de enseñanza sobre los algoritmos de la división de fracciones.

En un primer acercamiento, los análisis teóricos que se hacen para describir o elaborar modelos de enseñanza tienen como objetivo general identificar elementos fundamentales de: i) los contenidos matemáticos en juego, aspecto que se vincula con el componente de los Modelos de competencia formal del MTL; ii) la estructura del modelo, tanto conceptual, como didáctica, y iii) otras características relacionadas con las formas de comunicar las ideas matemáticas, aspecto asociado al componente de los Modelos de comunicación del MTL.

Entre los análisis teóricos con los cuales se han caracterizado modelos de enseñanza para la construcción de un MTL, en la línea de investigación del PNA de Valencia mencionada al inicio de este documento, desempeñan un papel esencial las metodologías de análisis didáctico e histórico-epistemológico de los libros de texto tanto históricos, como actuales.

El Análisis Didáctico es un marco metodológico para la investigación en educación matemática, que tiene rasgos particulares y admite diversos componentes y enfoques funcionales (para más detalle ver Rico, Lupiáñez y Molina, 2013). Uno de éstos es el análisis de contenido, que se refiere a los aspectos específicos relacionados con la estructura conceptual, los aspectos fenomenológicos o contextuales y los sistemas de representación.

La estructura conceptual se refiere a las ideas matemáticas, sus enlaces y relaciones; los aspectos fenomenológicos o contextuales son situaciones que están en el origen y dan lugar a los conceptos estudiados que les dan sentido; y las representaciones se usan para hacer presentes las nociones matemáticas.

El estudio descrito en este artículo se ha centrado en el análisis de contenido. Con él se ha pretendido identificar y describir aquellos aspectos específicos que se considera que forman parte de los elementos constitutivos de los modelos de enseñanza de los algoritmos de la división de fracciones. Este análisis se sustenta en el análisis histórico-epistemológico con el fin de rastrear la forma cómo estos elementos han estado presentes en diferentes momentos de la historia.

Dado que, de acuerdo con el MTL el estudio es local, en el sentido de que sólo pretende explicar los fenómenos que se ponen bajo observación, el trabajo se particulariza en identificar los tipos de algoritmos que han sido los protagonistas principales en los libros de texto de matemáticas, cuáles han sido sus fundamentos y cuál es el tipo de representaciones empleadas para esas explicaciones.

\subsection{Los periodos históricos y libros de texto}

Cuando el análisis es histórico-epistemológico, en tanto análisis de la formación de los objetos matemáticos a lo largo de su historia, se necesita de un enfoque de aproximación global, ya que estudiar textos de un momento aislado, o comparar varios textos entre sí de ese momento, es insuficiente, en la medida que tiende a desconsiderar las raíces y fuentes de las concepciones vertidas en el texto, su contexto social y cultural, y las particularidades propias del sistema educativo (Schubring, 
1987). Es en este sentido en el que adquiere importancia revisar una buena selección de libros de texto representativos de las grandes etapas en que se puede dividir la historia de las ideas matemáticas objeto de estudio, en función de hechos históricos y científicos decisivos y con influencia en las matemáticas de su época (Gómez, 2011a).

En el trabajo pionero realizado a principios de los noventa por Gómez (1994), se consideraron dos grandes puntos históricos de inflexión para los libros de texto, uno es la aparición de la imprenta a finales del siglo XV, y otro es el establecimiento del sistema general y público de enseñanza de corte estatal y centralista, al finalizar la revolución francesa en 1789.

Estos dos hechos históricos determinan tres grandes periodos que se pueden subdividir atendiendo a las especificidades de la investigación. Otros autores aportaron subdivisiones de estos grandes periodos. Así, por ejemplo, Sierra, González y López (1999) toman como referentes para agrupar los libros de texto las disposiciones legales, dado que los libros de texto tienen que adaptarse a ellas. Picado y Rico (2011a y 2011b) se centran en las leyes que promulgan y fijan la obligatoriedad del SMD (sistema métrico decimal) en la segunda mitad del siglo XIX y Maz (2005) se fija en los cambios político-sociales para seleccionar y agrupar los textos de matemáticas en España en un periodo determinado por los siglos XVIII y XIX.

Estos criterios han sido útiles para los objetivos de las investigaciones realizadas por el grupo de Historia y Educación Matemática de la SEIEM, y sirven como referencia para nuevos trabajos. Una combinación de los mismos permite organizar la producción española de libros de texto en los siguientes periodos y sub-periodos:

1. Periodo del libro impreso. En esta período se pueden distinguir los sub-períodos de las aritméticas comerciales (s. XV-XVI), del predominio de las órdenes religiosas en la enseñanza (s. XVI-XVIII) y del predominio de las instituciones civilesmilitares (s. XVIII-XIX).

2. Periodo del libro de enseñanza. En esta época, que arranca al comenzar el XIX, destacan los sub-períodos marcados por la ley Moyano de 1857 y por la obligatoriedad del sistema métrico decimal en 1892.

3. Periodo del libro escolar. En este periodo, que arranca con la implantación de la escuela graduada, son puntos de inflexión los primeros cuestionarios oficiales de 1953, el auge del estructuralismo y las matemáticas modernas (1970-1990) y el comienzo de la normalidad democrática en España (LOGSE en 1990).

Aunque no estrictamente, estos periodos han sido relevantes para efectuar la selección de libros que Mauricio usó en su análisis histórico-epistemológico de los problemas y algoritmos de división de fracciones. La información sobre estos últimos se verá reflejada en este artículo más adelante.

\subsection{Investigación precedente sobre la división de fracciones}

Sobre los algoritmos elementales de cálculo el currículo actual ofrece una perspectiva limitada que tiene consecuencias en el desempeño de los estudiantes. Esto es, la manera usual de enseñarlos tiene defectos de procedimiento y conceptuales que han sido puestos de manifiesto por medio de la investigación precedente realizada por diferentes autores. 
Según Newstead y Murray (1998), la división de fracciones fuera de contexto es una fuente de conflictos con las ideas de división de los estudiantes, que están muy enraizadas en la división entre números naturales. Afirman que uno de los errores más comunes consiste en ver la fracción como dos números enteros separados, en vez de identificarla como una cantidad o como una relación de cociente entre dos cantidades. Estos autores afirman que también en el caso de la división por una fracción, los estudiantes utilizan esquemas de los números enteros. Así ocurre, por ejemplo, cuando cambian $4 / 8$ por $8 / 4$, porque tienen el error conceptual de que la conmutatividad se puede aplicar tanto a la suma, como al cociente.

Yamaguchi y Jwasaki (1999) consideran que hay una separación entre el concepto de división y el algoritmo de división debido a que las nociones de repartir y medir, asociadas con los modelos semántico "partición" y "cuotición”, implicadas en las divisiones con números naturales no se pueden trasladar miméticamente a la división con fracciones. En su análisis afirman que la división con decimales es una especie de extensión de la división con números naturales; pero la división con fracciones no es una división, sino una multiplicación.

Consecuencia de todo esto es que el algoritmo de la división más asumido por los estudiantes, el de "invertir y multiplicar", se aprende de memoria, como una regla sin razones, ya que no se puede deducir de los conceptos de división previamente aprendidos con los naturales. Entonces, cabe preguntarse de qué otros conceptos se puede deducir el algoritmo de la división de fracciones y evitar así este tipo de aprendizaje memorístico.

Como propuesta metodológica, estos autores sugieren que el algoritmo "invertir y multiplicar" se puede deducir lógicamente y comprendiendo conceptualmente su significado haciendo referencia a propiedades de fracciones y reglas sobre la división, como por ejemplo recordando que "El valor de una fracción es constante cuando ambos, denominador y numerador se multiplican por el mismo número". Esto permite reducir a común denominador y convertir la división de fracciones en la división de sus numeradores, y por tanto en una división de enteros.

En este sentido, de usar las fracciones con el mismo denominador, Sharp (1998) parte del significado de división como resta repetida para construir el algoritmo de división de fracciones. Utiliza como soporte un modelo continuo circular de tarta con el que representa algunos casos particulares, primero con el mismo denominador: $3 / 4 \div 1 / 4$ (ver Figura 1) y luego con distinto denominador: $2 / 3 \div 2 / 6$. Incluye también el caso en que el resultado es menor que la unidad, como en $1 / 6 \div 3 / 6$. Del análisis de todos estos casos particulares, los estudiantes inducen el algoritmo de reducción a común denominador y división de los numeradores. 


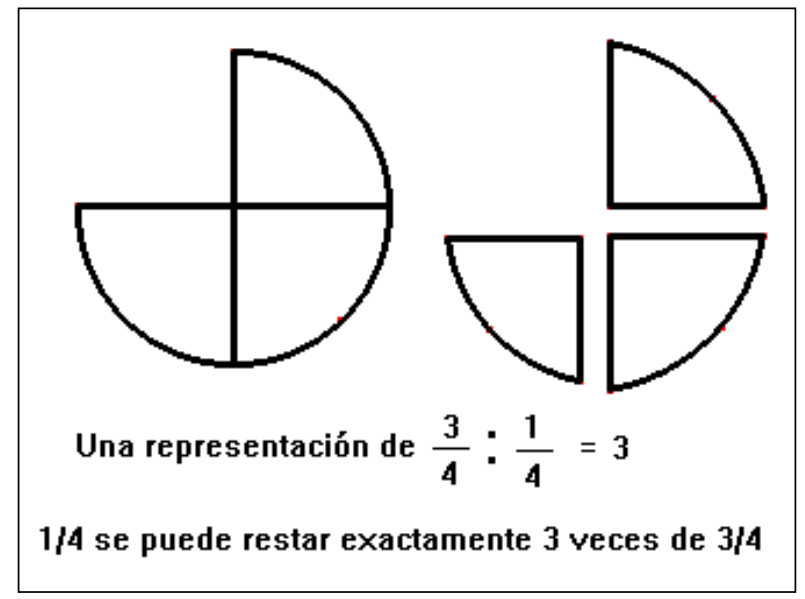

Figura 1. Representación de una división de fracciones con mismo denominador

Abundando en las formas de sustentar la división de fracciones, Sinicrope, Mick y Kolb (2002), tras afirmar que se puede explicar por extensiones de las tres interpretaciones de división de números enteros (medida, partición e inversión del producto cartesiano), aseguran que estas extensiones no son suficientes; consideran como necesarias la división como determinación de una razón unitaria y la división como la inversa de la multiplicación.

Una propuesta innovadora la hacen Brendefur y Pitingoro (2000), quienes utilizan un modelo de tabla de proporcionalidad como herramienta para representar la división de fracciones. De manera flexible, los estudiantes usan la equivalencia de razones para resolver problemas de división de fracciones en contexto. La situación propuesta es la siguiente: "Suponiendo que repartimos 1 1/4 cajas de cereal entre 4 personas, determina qué cantidad de cereal se lleva cada persona. Halla también qué cantidad de cereal se llevarían 60 personas". Algunos estudiantes descubren que 1 1/4 cajas dividido entre 4 personas es equivalente a 5 cajas dividido entre 16 personas. Usando razonamiento proporcional llegan a la cantidad de cajas de cereal para una sola persona (Figura 2):

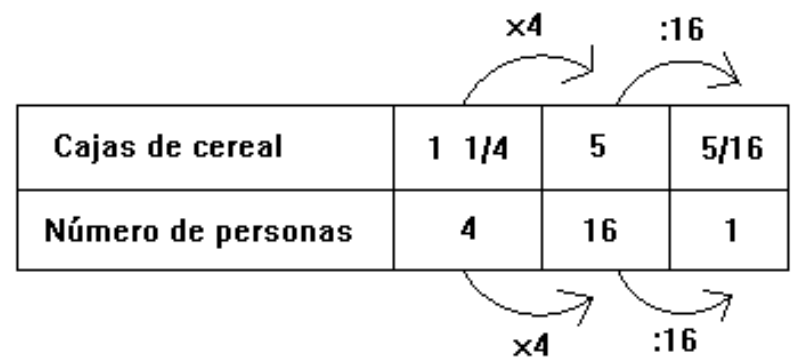

Figura 2. Relacionando la proporcionalidad con la división de fracciones

Por último, en la línea de deshacer la fracción, Warrington (1997) recomienda ayudar a los niños a que desarrollen sus propias estrategias de cálculo para dividir fracciones, sin que el profesor les muestre previamente algún algoritmo. Así, dice que cuando les propuso la división 4/2 los estudiantes dieron como resultado 2 , porque 2 cabe exactamente 2 veces en 4 . A la siguiente división: $2 \div 1 / 2$, respondieron 4 , ya que $1 / 2$ cabe exactamente 4 veces en 2 . En la siguiente: $1 \div 1 / 3$, los estudiantes contestaron 3 , porque $1 / 3$ cabe exactamente 3 veces en 1 . A $1 \div 2 / 3$, contestaron $1 \frac{1}{2}$, puesto que como $1 / 3$ cabe exactamente 3 veces en $1,2 / 3$ cabrá la mitad de veces, es decir $1 \frac{1}{2}$ veces. A la división $1 / 3 \div 3$ los estudiantes respondieron $1 / 9$, ya que hay que dividir cada tercio en tres partes iguales y cada una será 1/9. Al problema: "He comprado 5 3/4 
libras de chocolate. Quiero guardarlas en cajas de 1/2 libra cada una. ¿Cuántas cajas puedo hacer?", los estudiantes contestaron 11/1/2 cajas. El razonamiento fue: con 5 libras de chocolate se pueden llenar 10 cajas de $1 / 2$ libra. Con $3 / 4$ de libra se puede llenar otra caja de $1 / 2$ libra y queda $1 / 4$ de libra con el que se puede llenar media caja de $1 / 2$ libra. Este tipo de razonamiento se extiende hasta llegar a divisiones del tipo $42 / 5 \div 1 / 3$, que también son resueltas por algún alumno de forma similar, aunque introduciendo una partición. Así, 1/3 cabe exactamente 12 veces en 4 . Para dividir $2 / 5$ entre $1 / 3$, partimos cada quinto en tres partes iguales, con lo que la unidad queda dividida en 15 partes iguales. Entonces $2 / 5$ equivale a $6 / 15$ y $1 / 3$ equivale a $5 / 15$. Por tanto $1 / 3$ cabe exactamente 1 vez en $2 / 5$ y sobra $1 / 15$. Es decir, el resultado final es 13 1/15.

\section{Algoritmos}

A grandes rasgos se pueden distinguir entre algoritmos particulares y generales. Los primeros sólo permiten resolver algunos casos particulares de división de fracciones, pero que no son de aplicación universal, como ocurre con los segundos. A continuación se detallan los que se han encontrado en la revisión de los libros de texto. Primero se da cuenta de los algoritmos generales y después de los particulares.

\subsection{Reducción de las fracciones a común denominador y división de los numeradores}

Este algoritmo consiste en transformar las fracciones en otras equivalentes con el mismo denominador, reduciendo así la división de fracciones a la división de enteros, ya que solo hay que dividir los numeradores. Chuquet lo explica en el contexto numérico, con ejemplos concretos, y el tipo de representación que usa para el registro de acciones consiste en poner arriba y debajo de las fracciones dividendo y divisor los numeradores y el denominador común de las fracciones reducidas respectivamente:

$$
\frac{3}{4} \div \frac{2}{3} \rightarrow \frac{\begin{array}{l}
\frac{9}{3} \div \frac{8}{2} \\
12
\end{array}}{12}
$$

El texto literal de Chuquet (1484/1880) dice:

... para partir un número roto [una fracción] de esta manera se reduce el partidor [divisor] y el número a partir [el dividendo] si son diferentes [como en la reducción a común denominador] y después se divide el numerador por el numerador. Ejemplo, si se quiere partir un número roto por otro número roto como $\frac{3}{4}$ por $\frac{2}{3}$ reduce y hallarás 9 [numerador de la fracción equivalente a $\frac{3}{4}$ ], [pon] debajo $\frac{3}{4}$, y [hallarás] 8 [numerador de la fracción equivalente a $\frac{2}{3}$ ], [pon] debajo $\frac{2}{3}$. Después parte 9 por 8 y tendrás $1 \frac{1}{8}$, y quien quiera partir $\frac{2}{3}$ por $\frac{3}{4}$ deberá partir 8 entre 9 y obtendrá $\frac{8}{9}$.

$\frac{10}{\frac{2}{3}} \frac{12}{3}$ Ejemplo, quien quiera reducir $\frac{2}{3}$ y $\frac{4}{5}$ primero debe multiplicar 3 por 5 , que monta 15 ,

para tener el denominador común. Después parte 15 por 3 y tendrás 5 , multiplícalo por 2 y hallarás 10 , que son $\frac{10}{15}$, para $\operatorname{los} \frac{2}{3}$. Después parte 15 por 5 y luego multiplica por 4 , y hallarás 12 que son $\frac{12}{15}$ para los $\frac{4}{5}$ y se pueden poner así como se ve al margen (Ibid, p. 612).

El mismo planteamiento y contexto se encuentra en Pérez de Moya (1562), pero con una representación diferente, porque ahora las acciones a realizar para llegar al resultado final están completamente explicitadas. Obsérvese también el uso que hace 
de la cruz de San Andrés y de la caja de la división para hacer ostensiva una disposición práctica de los datos.

La primera diferencia es partir un quebrado solo por otro solo, como quien dijese, parte $\frac{2}{5}$ por $\frac{1}{6}$; lo cual harás asentando la partición, que son $\operatorname{los} \frac{2}{5}$, a la mano izquierda y el partidor, que es un sexto a la derecha, de la manera que parece

$$
5>_{6}^{1}
$$

Y hecho esto, reducirás (según mostré en cxiii de este segundo lib.) multiplicando en cruz, como las rayas muestran, no curando de multiplicar los denominadores. Y lo que estuviese sobre la partición, se partirá por lo que estuviese sobre el partidor, como parece

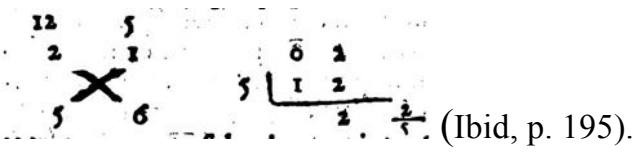

En cierto modo la propuesta de Yamaguchi lo que hace es volver a poner en la enseñanza este método. Nótese que en Chuquet, el modo de reducir a común denominador es el de multiplicar y dividir por la misma cantidad, lo que salvando las distancias equivale la recomendación de Yamaguchi cuando dice que hay que sustentar el algoritmo en las alteraciones invariantes de las fracciones.

\subsection{Multiplicar por la fracción inversa o recíproca de la fracción divisor}

Este algoritmo se enuncia diciendo que se multiplica el dividendo por el inverso del divisor. Así, para efectuar la división $\frac{a}{b} \div \frac{c}{d}$, se procede de la siguiente manera:

$$
\frac{a}{b} \div \frac{c}{d}=\frac{a}{b} \cdot \frac{d}{c}=\frac{a \cdot d}{b \cdot c}
$$

Este algoritmo se apoya en el concepto de fracción inversa, entendida como aquella que restaura a la unidad de una fracción dada. Esta idea está presente en la matemática árabe como prerrequisito para convertir la división de fracciones en multiplicación.

En el Liber Mahameleth (1200/2014, p. 726) encontramos la introducción de la fracción inversa bajo el siguiente razonamiento:

Those who divide a number by a number want to know what will be attributed to one, as we said before in the division of integers, whether the division is made by less or more than one. Now it is useful for those who want to divide fractions to know two things. The first is to know the number by the multiplication of which a fraction is reintegrated into one, the other is to know what ratio the unit has to an integer with a fraction.

Esta frase "un entero con una fracción" se refiere a un número mixto, ya que la inversa de una fracción, que en esa época era entendida como un número menor que uno, es necesariamente un número mayor que uno, que no era considerado fracción y por lo tanto se expresaba como un entero más una fracción propia. Por tanto, en términos actuales, lo que quiere decir la frase completa es que lo que hay que saber es la razón que tiene la unidad con una fracción impropia.

A continuación en el Liber Mahameleth se explican estas ideas con varios ejemplos. Primero con fracciones unitarias y después con fracciones propias.

That by which a fraction is reintegrated is like asking: [Cuál es el número por el cuál un tercio debe multiplicarse para restaurar la unidad] What is the number by which a third when multiplied is reintegrated into one? We shall answer: the multiplication of it by three does it. For there are three 
thirds in one, and the above third is a third of one; multiplying it by three produces one. A fourth is itself reintegrated by multiplying it by four, a half likewise by multiplying it by two, a sixth by multiplying it by six.

Someone then asks: What is the number by which two thirds are to be multiplied to produce one? Say that the multiplication of them by one and a half does it. For there are in one three thirds, and two thirds are as if it were two; then two, to produce three, will be multiplied by one and a half. Likewise, three fifths are reintegrated into one by multiplying them by one and two thirds; five sevenths are reintegrated into one by multiplying them by one and two fifths; and, similarly, eight elevenths by multiplying them by one and three eighths.

Obsérvese que en el Liber Mahameleth no se toma 5/3 como fracción inversa de $3 / 5$, que es lo que haríamos hoy, sino que se toma $1+2 / 3$, ya que al ser $5 / 3$ mayor que uno, no se consideraba fracción sino número mixto.

El mismo algoritmo de la fracción inversa se encuentra también en las aritméticas comerciales del siglo XVI, como se puede ver en el siguiente ejemplo de Stifel (1544), donde de manera explícita ya se menciona que por esta regla la división se reduce a invertir y multiplicar:

La regla para dividir se reduce a la regla para multiplicar minucias, de este modo: conmuta los términos a dividir, esto es pon el numerador debajo de la raya, y el denominador ponlo encima, y que cuando eso está hecho, ya no hay que hacer nada sino operar de acuerdo con las reglas dadas antes de la multiplicación (Ibid, p.6).

En el ejemplo que usa Stifel para ilustrar el método, aparecen las fracciones impropias y la representación simbólica de la fracción inversa, aunque finalmente transforma la fracción impropia 4/3 en el número mixto 11/3.

El mismo algoritmo lo encontramos incluso en los manuales escolares del comienzo de la época estructuralista, como se muestra en el siguiente ejemplo del año 1969.

Para dividir dos fracciones se multiplica la primera por la segunda invertida.

Ejemplo: $\frac{5}{3} \div \frac{4}{7}=\frac{5}{3} \times \frac{7}{4}=\frac{35}{12}$ (Valdivia y García, 1969, p. 38).

\subsection{Productos cruzados}

El algoritmo de los productos cruzados consiste en multiplicar los numeradores con los denominadores siguiendo el esquema de la cruz de San Andrés, como se muestra en la Figura 3.

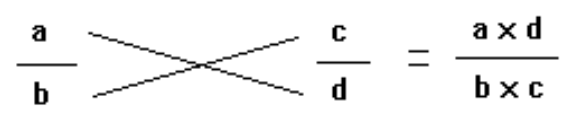

Figura 3. Representación del algoritmo productos cruzados

Puede entenderse que se trata de la regla que se deriva del algoritmo de reducir a común denominador, pero también se deriva del algoritmo de multiplicar por el inverso del divisor. Sin embargo en la literatura aparece como un método distinto de los anteriores, que se conoce como el método de los productos cruzados, por eso aquí solo se da cuenta de él con un ejemplo tomado de Andrés de Zaragoza (1515) del siglo XVI.

Obsérvese el énfasis que pone Andrés de Zaragoza en la representación de la disposición práctica de los datos, y que no hace mención, a diferencia de Pérez de 
Moya, en el fundamento de la misma. El contexto es numérico y la regla se describe a través de ejemplos concretos.

$\frac{1}{2}>\frac{2}{3} \quad \begin{gathered}\text { práctica de } \\ \text { la figura }\end{gathered} \frac{1}{2}>\frac{2}{3} \frac{3}{4}$

La cual se hace poniendo siempre la suma partidera [dividendo] hacia la mano sinistra y el partidor [divisor] hacia la mano derecha y multiplicando así como la precediere hallarás 4 encima del brazo derecho por partidor y hallaras sobre el brazo sinistro 3 por suma partidera que es menor que el partidor, y por ello has de poner el partidor que es cuatro debajo de una raya y pondrás la suma partidera que es 3 encima de la raya y nombrarás la suma partidera por el nombre del partidor diciendo tres quartos y ya tenemos la particion entegral y nominal declaradas (Andrés de Zaragoza, 1515, Capítulo 5 del tercer tratado fo lxvi).

\subsection{Deshacer la fracción por reducción a la unidad fraccionaria}

Este algoritmo consiste en deshacer la fracción divisor con un razonamiento de tipo proporcional en dos pasos que es similar al método de reducción a la unidad, en este caso la reducción es a la unidad fraccionaria. Una vez conocido el valor de la fracción unitaria se obtiene el valor de la unidad total que se pide en el enunciado con un razonamiento de tipo inverso al del primer paso. El siguiente ejemplo sirve para ilustrar el razonamiento.

Se han comprado $\frac{3}{8}$ de $\mathrm{Hg}$ de azafrán por $\frac{7}{9}$ de peseta. ¿A cuánto resulta el Hg? Sol: $\mathrm{Si} \frac{3}{8}$ valen $\frac{7}{9}$ de pta, $\frac{1}{8}$ vale $\frac{7}{9 \times 3}$ y $\frac{8}{8}$ valen $\frac{7 \times 8}{9 \times 3}=\frac{56}{27}$, o bien $\frac{7}{9}: \frac{3}{8}=\frac{56}{27}=2 \frac{2}{27}=2,074$ ptas. (Dalmau Carles, 1898, p. 152).

\subsection{Algoritmos producto de cancelaciones}

Frisius (1582, p. 41), después de introducir el producto en cruz como algoritmo general, menciona los siguientes casos particulares:

- Los denominadores son iguales. El algoritmo se reduce a la división de los numeradores, como se puede ver en el ejemplo $\frac{27}{32} \div \frac{2}{32}$ que da $\frac{9}{32}$.

- Los numeradores son iguales. Basta con poner el denominador del dividendo debajo del denominador del divisor, como por ejemplo $\frac{3}{4} \div \frac{3}{8}$, que da $\frac{8}{4}$, o 2 enteros. A los ojos de hoy esto es simplemente el resultado de multiplicar por la fracción inversa y cancelar el factor común.

- Uno de los numeradores contiene [múltiplo] al otro. Hay dos casos, que el numerador menor es el del divisor, entonces se halla el producto del denominador del menor por el número de veces que el mayor contiene al menor, y ese producto es el numerador de la fracción cociente, y su denominador es el denominador de la fracción con numerador mayor. El otro caso es que el numerador menor es el del dividendo, entonces es a la inversa, el producto (del número de veces que el mayor contiene al menor por el denominador del numerador menor) es el denominador y el numerador es el denominador del numerador mayor. Así, por ejemplo, si se trata de dividir $\frac{3}{5} \div \frac{12}{13}$, como 3 está contenido 4 veces en 12 multiplica 5 por 4 , que da 20 y este es el denominador, y 13 el numerador, o sea $\frac{13}{20}$. Al contrario, si la 
división es $\frac{12}{13} \div \frac{3}{5}$, se tendrá $\frac{20}{13}$.

- Forcadell (1585, p. 57 dcha.) en los comentarios a las reglas de las fracciones de Frisius, añade también el caso de que ambos numerador y denominador de una fracción sean múltiplos de los respectivos numerador y denominador de la otra. En ese caso el algoritmo consiste en dividir los numeradores y denominadores entre sí. Como, por ejemplo, en $\frac{16}{27}$ dividido por $\frac{2}{3}$ que da $\frac{8}{9}$, porque 16 dividido por 2 da $8 \mathrm{y}$ 27 dividido por 3 da 9 , que hacen $\frac{8}{9}$.

\subsection{Conversión de fracciones en decimales}

Este algoritmo consiste en transformar las fracciones en decimales por división, se hace la operación indicada y finalmente se expresa el resultado volviendo a la forma de fracción. El siguiente ejemplo, corresponde a la época de abandono de las matemáticas modernas y regreso a lo básico, en el marco de la reforma educativa de la LOGSE.

Ejemplo: $\frac{1}{2} \div \frac{3}{4}=0,5 \div 0,75=\frac{50}{75}=\frac{2}{3}$ (Hernán y Carrillo, 1988, p. 164).

En este método la división de dos fracciones se convierte en una sola fracción por una cadena de equivalencias, como se ve en el ejemplo que pone el autor no se opera, solo se usan diferentes maneras de representar simbólicamente las fracciones, obteniendo así el resultado directamente. Este método funciona porque las fracciones utilizadas son fracciones decimales finitas, y no funcionaría para dividir $1 / 3$ entre $2 / 7$, que no son finitas sino periódicas. Por tanto es un método particular que sólo funciona con fracciones específicas.

\section{Fundamentos para el algoritmo}

En la revisión de textos realizada se observa que los autores intentan fundamentar los algoritmos de la división de fracción por extensión de los modelos de dividir con naturales, principalmente con los modelos de "cuotición" e inversión de la multiplicación. Recordemos que al pasar a fracciones, el modelo de reparto no tiene sentido (la estrategia de partir o repartir el dividendo no puede llevarse a cabo cuando el divisor es una fracción), y que aparecen nuevos modelos como el denominado "parte de" y el Euclídeo: $\frac{D}{d}=\frac{q}{1}$ o $\frac{D}{q}=\frac{d}{1}$

Bajo este modelo (parte de) el significado de los términos cambia. Ahora, multiplicar es tomar de un número dado una parte denotada por el multiplicador, el multiplicador es la parte o fracción que se toma y el multiplicando es la cantidad de la que se toma una parte. En la división, cuando se toma como divisor el multiplicador, el divisor es la fracción o parte que se toma, el dividendo es la parte resultante, y el cociente es la cantidad de la que se toma una parte, que es la cantidad buscada (Gómez, 2011b, p. 55).

A continuación se da cuenta de cómo los autores de los textos de los diferentes periodos revisados intentan fundamentar los algoritmos de la división de fracción a la luz de los distintos modelos de la división disponibles.

\subsection{Interpretación de la división como "cuotición”.}

Para explicar el algoritmo de deshacer la fracción, algunos textos como por ejemplo Bails (1818) y Edelvives (1934), se apoyan en la interpretación cuotitiva de la 
división, donde de lo que se trata es de averiguar cuántas veces cabe el divisor en el dividendo, o lo que es lo mismo, cuántas veces contiene el dividendo al divisor. En ambos textos se usa el método de deshacer la fracción, llama la atención que ya en la época de Bails (la primera edición del final del siglo XVIII) se usará un método razonado, que en palabras de investigadores como Warrington los niños son capaces de descubrirlos para casos concretos.

La razón de esta regla es, que partir $\frac{4}{5}$ por $\frac{2}{3}$ es buscar quantas veces $\frac{2}{3}$ cabe en $\frac{4}{5}$; pero se viene a los ojos que pues el divisor expresa tercios, cabrá en el dividendo tres veces más que si expresara enteros, luego se ha de dividir primero por 2, y multiplicar después por 3, lo mismo cabalmente que tomar tres veces la mitad del dividendo, o multiplicar por $\frac{2}{3}$ (Bails, 1818, p. 63).

En el texto de Edelvives (1934), se desglosa la explicación en dos casos: en el primero se aplica a la división por la fracción unitaria y en el segundo a un múltiplo de la misma.

Dividir 9 por $\frac{1}{4}$.- Esto equivale a hallar las veces que 9 contiene a $\frac{1}{4} ; 1$ unidad lo contiene 4 veces; 9 unidades lo contendrán $9 \times 4=36$ veces. La aplicación de la regla da este mismo resultado:

$$
9: \frac{1}{4}=9 \times \frac{4}{1}=\frac{36}{1}=36
$$

Dividir 9 por $\frac{3}{4}$ - - Esta división difiere de la anterior en que el divisor es triple; el dividendo lo contendrá, pues, 3 veces menos, o sea $\frac{36}{3}=12$. Es lo que resulta al aplicar la regla:

$$
9: \frac{3}{4}=9 \times \frac{4}{3}=\frac{36}{3}=12(\text { Ibid, p. 129-130). }
$$

\subsection{Interpretación de la división como "parte de"}

En el texto de Rey Pastor y Puig Adam (1932) se explica el algoritmo de reducir a común denominador en términos de cambio de unidad fraccionaria. En los datos aparecen las unidades fraccionarias, novenos en el dividendo y séptimos en el divisor, por ello para poder averiguar qué parte del divisor es el dividendo se busca una unidad fraccionaria común: sesenta y tres avos: $\frac{m}{n} \times \frac{3}{7}=\frac{2}{9}$.

Si cada torta pesa $\frac{3}{7}$ de kilo, ¿qué porción de torta tendré con $\frac{2}{9}$ de kilo? Indicaremos la operación así: $\frac{2}{9} \div \frac{3}{7}$ (dividendo y divisor homogéneos). Reduciendo los pesos a la misma parte alícuota de $\mathrm{kg}$ plantearemos la pregunta de este otro modo: Si cada torta pesa $\frac{3 \cdot 9}{7 \cdot 9}$ kilogramos, ¿cuánto tendré por $\frac{2 \cdot 7}{9 \cdot 7}$ ? De modo que, tomando por nueva unidad $\frac{1}{7.9} \mathrm{~kg}$, la torta pesa 3.9 unidades, luego con $2 \cdot 7$ unidades tendré una porción de torta igual a $\frac{2 \cdot 7}{3 \cdot 7}$. (Ibid, pp. 211 y 212 ).

\subsection{Interpretación de la división desde el modelo Euclídeo}

Lacroix (1825) explica el método de invertir el divisor desde la interpretación Euclídea de la división $\frac{D}{q}=\frac{d}{1}$; esto es, que la relación del dividendo al cociente es la misma que la del divisor a la unidad.

La palabra "contiene", en su sentido estricto, ya no es apropiada para todos los casos que se presentan en la división, igual que la palabra "repetido en todos aquellos presentados en la multiplicación; por eso no se puede decir que el dividendo contiene al divisor, cuando este es 
menor que aquél, [aunque] la expresión se usa generalmente, pero solo por analogía y extensión.

Para generalizar la división, "el dividendo debe considerarse como teniendo la misma relación con el cociente que el divisor con la unidad", porque el divisor y el cociente son los dos factores del dividendo. Esta consideración es conforme para todos los casos en que la división puede presentarse. Cuando, por ejemplo, el divisor es 5, el dividendo es 5 veces el cociente, y, consecuentemente, el cociente es la quinta parte del dividendo. Si el divisor es una fracción, $1 / 2$ por ejemplo, el dividendo no puede ser más que la mitad del cociente, y este último debe ser el doble del primero. regla.

Después explica porque eso equivale a multiplicar por el factor inverso, que es la

La definición, que se acaba de dar, fácilmente sugiere el modo de proceder, cuando el divisor es una fracción. Sea, por ejemplo $\frac{4}{5}$. En este caso el dividendo debe ser solo $\frac{4}{5}$ del cociente; pero como $\frac{1}{5}$ es $\frac{1}{4}$ de $\frac{4}{5}$, tendremos $\frac{1}{5}$ del cociente, tomando $\frac{1}{4}$ del dividendo, o dividiendo por 4 . Ahora, teniendo $\frac{1}{5}$ del cociente, solo tenemos que tomarlo 5 veces, o multiplicarlo por 5 , para obtener el cociente. En esta operación el dividendo se ha dividido por 4 y multiplicado por 5, lo que es lo mismo que tomar $\frac{5}{4}$ del dividendo, o multiplicarlo por $\frac{5}{4}$, fracción que no es otra que el divisor invertido (Ibid, p. 47 y 48).

\subsection{Interpretación de la división como inversión de la multiplicación en el modelo "factor faltante": $D=d q$.}

Fernández y Cardín (1863) explican el algoritmo de multiplicar por el inverso del divisor empleando la relación multiplicativa entre los distintos elementos de la división: $D=d q$. En esta relación el cociente $q$ es interpretado como el factor faltante, $\mathrm{y}$ éste debe ser el producto del dividendo por el inverso del divisor porque es el que cumple con la relación, ya que $d \times D \frac{1}{d}=D$

Sea dividir $\frac{2}{3}$ por $\frac{5}{7}$, como el cociente multiplicado por el divisor da el dividendo, dicho cociente es el producto del dividendo por el divisor invertido, esto es $\frac{2}{3} \times \frac{7}{5}$; pues este producto multiplicado por $\frac{5}{7}$ es igual al dividendo $\frac{2}{3}$; en efecto. $\frac{2}{3} \times \frac{7}{5} \cdot \frac{5}{7}=\frac{2}{3} \times 1=\frac{2}{3}$. Luego para dividir un quebrado por otro se multiplica el dividendo por el divisor invertido, o lo que es igual, se multiplica el numerador del dividendo por el denominador del divisor, y el denominador del dividendo por el denominador del divisor, siendo el primero de estos productos el numerador y el segundo el denominador del cociente buscado (Ibid, p. 63).

En los libros recientes, como por ejemplo los de García Roca (1965) y Bujanda y Mansilla, 2002), se mantiene este tipo de argumentación. Incluso, se puede encontrar una sustentación del algoritmo en un mayor nivel de generalización, en términos de la solución de una ecuación (Rey Pastor y Puig Adam, 1932, p. 37 y Rey Pastor, Pi Calleja y A. Trejo, 1957, p. 69; Valdés y Santos, 1975, p. 40-41).

\subsection{Interpretación de la división como inversión de la multiplicación en el modelo "factor multiplicativo" (hacer tantas veces mayor o menor)}

Para explicar el algoritmo de los productos cruzados, Vallejo (1841) considera que cuando el divisor es una fracción, la división es hacer el dividendo tantas veces menor como indica el numerador del divisor $y$ tantas veces mayor como indica el denominador del divisor. 
Para dar la demostración de esta regla nos contraeremos al primer ejemplo [dividir $\frac{3}{4}$ por $\frac{2}{5}$ ]; y observaremos que si solo tuviésemos que dividir por 2 , numerador del divisor $\frac{2}{5}$, estaba reducida la operación á hacer dos veces menor el quebrado, lo que se consigue multiplicando su denominador por 2, de manera que $\frac{3}{8}$ sería el cociente; pero nosotros no teníamos que dividir por 2 , sino por $\frac{2}{5}$, que es cinco veces menor que el verdadero; y así, para obtener este, debemos hacer aquel cinco veces mayor; lo cual se consigue multiplicando su numerador por 5; de manera que ejecutándolo, tendré por cociente verdadero $\frac{15}{8}=1 \frac{7}{8}$ (Ibid, p.120).

\subsection{Alteraciones invariantes de la división}

Vallejo (1841) también justifica el algoritmo de reducir a común denominador desde las propiedades de la división, concretamente desde sus alteraciones invariantes, lo que le permite deducir la regla es multiplicar en cruz, evitando cualquier interpretación de la división.

Esta regla la podríamos deducir analíticamente con bastante sencillez y claridad, observando que como en la división los términos deben ser homogéneos, para ejecutar esta operación deberemos hacer que lo sean los quebrados, reduciéndolos a un común denominador; de manera que yendo indicando la operación tendremos, $\frac{3}{4}: \frac{2}{5}=\frac{3 \times 5}{4 \times 5}: \frac{2 \times 4}{5 \times 4}$. Ahora, como un cociente no se altera, aunque se multipliquen o partan el dividendo y divisor por un mismo número, resulta que podremos multiplicar los dos términos de la división indicada arriba por el denominador $4 \times 5$; y como esto se consigue suprimiendo los denominadores se tendrá $3 \times 5: 2 \times 4$, o indicando la división por medio de la raya será $\frac{3 \times 5}{2 \times 4}$. Resultado que manifiesta que se ha de ejecutar la multiplicación en cruz para obtener directamente el cociente (Ibid, p. 120).

Igualmente, Anzola y Vizmanos (1997) utilizan las alteraciones invariantes de la fracción para generalizar el caso particular descrito por Forcadell (ver apartado anterior) -en el cual el numerador y el denominador del dividendo son múltiplos de los del divisor- a las fracciones donde no se da esta condición. El método consiste en hacer que el numerador y el denominador del dividendo sean múltiplos del numerador y el denominador del divisor, y para ello basta con multiplicar los primeros por el producto de los segundos.

Obteniendo una fórmula general: si el numerador y el denominador del dividendo no son múltiplos de los del divisor, se procede así para obtener la regla que se utiliza normalmente: $\frac{3}{5}: \frac{4}{7}=\frac{3 \cdot 4 \cdot 7}{5 \cdot 4 \cdot 7}: \frac{4}{7}=\frac{3 \cdot 7}{5 \cdot 4}=\frac{21}{20}$ (Ibid, p. 25).

Contreras (2007, pp. 149-150) presenta una variante de este algoritmo, usando conjuntos discretos (bolas). Él evita interpretar la división usando la alteración invariante que resulta al multiplicar los términos de la división por un mismo número, concretamente el denominador común: $\frac{3}{4} \div \frac{3}{5}=\frac{3}{4} \times 20=\frac{3}{5} \times 20=15 \div 12=\frac{5}{4}$ (Figura 4). Obsérvese el uso que hace de la equivalencia entre las formas de representación fracción y cociente indicado.

¿Cuál es el resultado de la división $\frac{3}{4} \div \frac{3}{5}$ ? Tomamos tantas bolas como indica el producto de los denominadores, es decir, 20. Hay que dividir tres cuartos de las 20 bolas (es decir, 15 bolas) entre los $3 / 5$ de 20 bolas (es decir, 12 bolas). Por tanto, el resultado de la división es $\frac{15}{12}=\frac{5}{4}$ de las bolas. Así: 


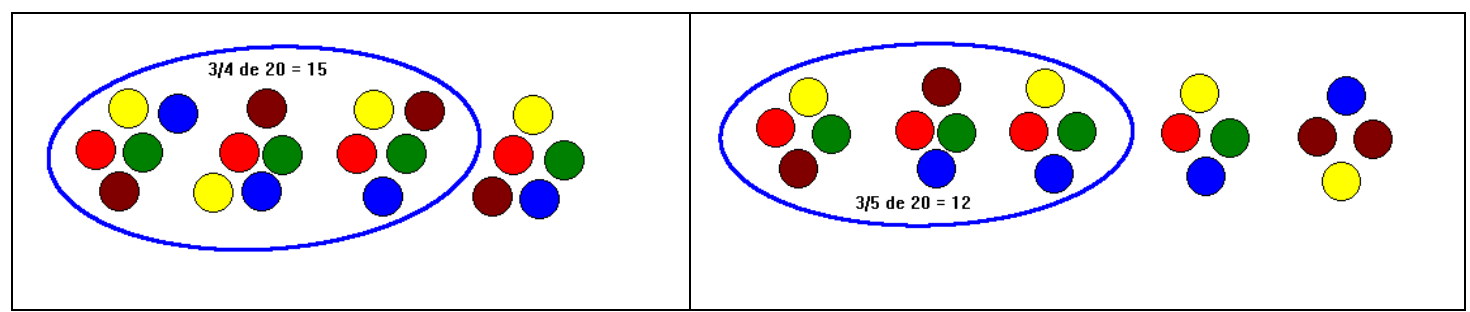

Figura 4. Representación de la división de fracciones (Ibid, pp. 149-150)

\subsection{Interpretación de la división desde el modelo de área}

Las figuras rectangulares son las representaciones más socorridas para explicar el algoritmo "parte de" de la división usando el modelo de área, donde se trata de hallar uno de los lados conocido el otro y el área.

Anzola y Vizmanos (1995) aplican esta representación para deducir el algoritmo de Forcadell:

En este primer rectángulo se tiene:

El área del rectángulo mide 35.

La base mide 7 .

La altura del rectángulo medirá 35:7 =5.

En este segundo rectángulo se tiene:

El área del rectángulo mide $\frac{15}{35}$.

La base mide $\frac{5}{7}$.

La altura del rectángulo medirá $\frac{15}{35}: \frac{5}{7}$

Por otra parte, esta altura mide $\frac{3}{5}$.

Si estos dos procesos tienen que dar el mismo resultado, debe ser:

$$
\frac{15}{35}: \frac{5}{7}=\frac{15: 5}{35: 7}=\frac{3}{5} \text { (Ibid, p. 25). }
$$

Ott, Snook y Gibson (1991) también usan este modelo, aunque en el caso en que el denominador del dividendo es múltiplo del denominador del divisor, como se puede ver en el siguiente ejemplo:

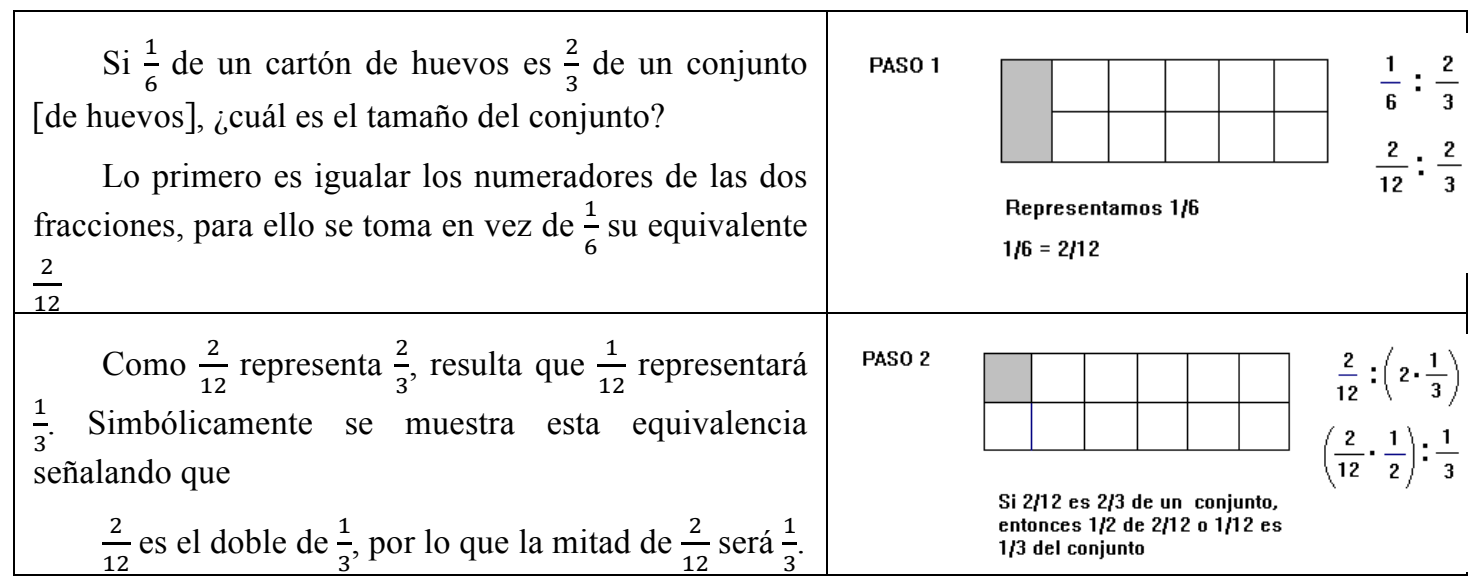




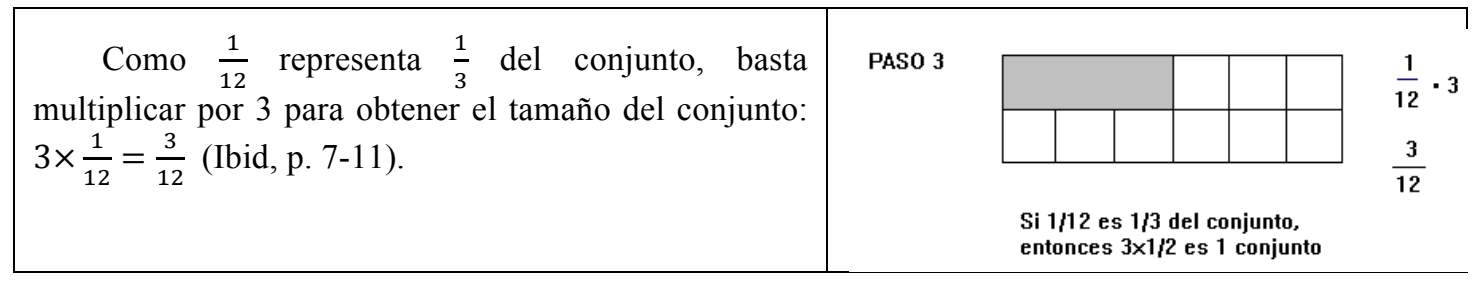

Sinicrope, Mick y Kolb (2002) también aplican este modelo para explicar el caso particular del algoritmo de Forcadell. El razonamiento es como sigue:

Supóngase que se busca la anchura del
rectángulo conociendo su área (6/20) y su altura
(3/4), el primer paso es representar la partición de
la altura en "cuartos".

\section{Epílogo}

En los apartados anteriores se ha dado cuenta de una parte del análisis didáctico de la división de fracciones. En particular de la estructura conceptual, en la componente análisis de contenido, reflejada en los libros de texto de los algoritmos de la división de fracciones, desde un acercamiento histórico-epistemológico. Este análisis, ha permitido mostrar los distintos algoritmos de la división de fracciones vigentes o presentes en la tradición de enseñanza: reducir a común denominador, productos cruzados, multiplicar por el inverso del divisor y deshacer la fracción, También se ha dado cuenta de las formas y representaciones usadas para sustentar las explicaciones de los mismos que los autores de esos textos han preferido. Incluso, a la vista de la información obtenida, se podría pensar en relaciones entre interpretaciones de la división y algoritmos, dado que los autores muestran preferencias al ligar 'cuotición' con 'deshacer la fracción'; 'parte de' con 'reducción a común denominador' y 'factor faltante' con 'multiplicar por el inverso del divisor', y también que las alteraciones 
invariantes de la división se usan para evitar tener que dar una interpretación de la división para las fracciones.

Se podría decir que las ideas que los investigadores en educación matemática proponen para la enseñanza de la división de fracciones no son nuevas, sino son opciones que ya están presentes en los libros desde hace mucho tiempo.

En consecuencia, el análisis de los libros de texto de matemáticas antiguos y actuales, debe ser considerado como una valiosa fuente de inspiración para el diseño de modelos de enseñanza, para la formación de profesores, los diseñadores del currículo y los escritores de los libros de matemáticas escritos para su enseñanza.

Por último, se quiere señalar que en este artículo, no solo se recogen las aportaciones de Mauricio Contreras para un modelo de enseñanza sobre los algoritmos de la división de fracciones en el marco de los MTL; sino que, se ha reformulado la exposición global de sus ideas y se ha ampliado la revisión de textos. Con ello se ha pretendido dar a conocer lo que Mauricio estaba intentando llevar adelante y que no pudo terminar.

\section{Referencias}

Andrés de Zaragoza, Juan (1515). Sumario breve de la práctica de la arithmética y todo el curso de larte mercantinol bien declarado: el qual se llama maestro de cuento. Valencia: Impresor Juan Joffre.

Anzola, M. y Vizmanos, J. R. (1995). Matemáticas $3^{\circ}$ ESO. Madrid: SM.

Bails, B. (1818). Principios de Matemática de la Real Academia de San Fernando. Imprenta de Joaquín Ibarra. Quinta edición. Tomo I.

Brendefur, J. L. y Pitingoro R. C. (2000). Dividing fractions by using the ratio table. En Lorna, J. Morrow and Margaret, J. Kenney (Eds.), NCTM 1998 Yearbook: The teaching and learning of algorithms in school mathematics, pp. 204-207. Reston, VA: NCTM.

Bujanda, M. P. y Mansilla, S. (2002). Números. Matemáticas $2^{\circ}$ ESO. Madrid: SM.

Chuquet (1484). Triparty. En Marre, A. (Ed.), 1880. Notice sur Nicolas Chuquet et son Triparty en la science des nombres. Bulletino di Bibliografia e di Storia delle Scienze Matematiche e Fisiche Publicato da B. Boncompagni 13, 555-659, 693-814.

Contreras, M. (2012). Problemas multiplicativos relacionados con la división de fracciones. Un estudio sobre su enseñanza y aprendizaje. Tesis doctoral. Valencia: Universidad de Valencia.

Contreras, M. (2007). Matemáticas $3^{\circ}$ ESO. Valencia. Edición del autor.

Dalmau Carles, J (1898). Soluciones analíticas de los ejercicios y problemas contenidos en los libros "Aritmética razonada y nociones de Álgebra" y "Lecciones de Aritmética”. Madrid: Librerías Hernando e Hijos de Pérez. (Reedición de 1943).

Edelvives (1934). Aritmética de Segundo grado. Zaragoza. Séptima edición.

Fernández y Cardín, J. M. (1863). Elementos de Matemáticas. Madrid: Impreso por Gómez Fuentenebro, A. Cuarta edición. 
Filloy, E.; Puig, L. y Rojano, T. (2008). Educational Algebra. A Theoretical and Empirical Approach. N. Y.: Springer

Forcadel, P. (1585). Commentaires sur les règles des fractions . En L'Arithmetique de Gemme Phrison. Traduite an François par Pierre Forcadel de Beziers, Professeur des Mathematiques, par luy ilustrée de Commentaires, contenans plusieurs inventions nouvelles du dit Forcadel. Augmentee en cette derniere edition des Commentaires sur les règles des fractions. Paris. Chez Hierosme de Marnee.

Frisius, G. (1582). L'Arithmetique de Gemme Phrison. Traduite an François par Pierre Forcadel de Beziers, Professeur des Mathematiques, par luy ilustrée de Commentaires, contenans plusieurs inventions nouvelles du dit Forcadel). En Anvers, chez Iean Vithage.

García Roca (1965). Matemáticas $1^{\circ}$ curso. Valencia: Bello.

Gómez, B. (1994). Los métodos de cálculo mental en el contexto educativo: un análisis en la formación de profesores. Tesis doctoral. Universidad de Valencia.

Gómez, B. (2011a). Marco de referencia para contextualizar la investigación en Historia y educación matemática. Epsilon, 1 (LXIII), 110-115.

Gómez, B. (2011b). Discontinuidad de los modelos de situación de las operaciones multiplicativas. Educatio siglo XXI, vol 29, n², pp 41-66.

Hernán, F y Carrillo, M. (1988). Recursos en el aula de matemáticas. Matemáticas: cultura y aprendizaje. Madrid: Síntesis.

Lacroix, S. F. (1825). An elementary Treatise on Arithmetic taken principally from the Arithmetic of S. F. Lacroix, and translated from the French with such alterations and additions as where found necessary in order to adapt it to the use of American students. By John Farr, Third edition, Cambridge: Hilliard and Metcalf. University Press.

Maz, A. (2005). Los números negativos en España en los siglos XVIII y XIX. Tesis doctoral. Universidad de Granada, España.

Newstead, K. y Murray, H. (1998). Young Students' Constructions of Fractions. En A. Olivier y K. Newstead (Eds.). Proceedings of the $22^{\text {nd }}$ Conference of the international Group for thePME, 3, 295-303, Sudáfrica.

Ott, J. M., Snook, D. L. y Gibson, D. L. (1991). Understanding partitive division of fractions. Arithmetic Teacher, 39(2), 7-11.

Pérez de Moya, J. (1562). Arithmetica práctica y speculativa. Salamanca. Biblioteca Castro. Madrid: Ediciones de la Fundación José Antonio de Castro. 1998.

Picado, M. y Rico, L. (2011a). Análisis de contenido en textos históricos de matemáticas. PNA, 6(1), pp. 11-27.

Picado, M. y Rico, L. (2011b). La selección de textos en una investigación histórica en Educación Matemática. Épsilon, 28(1), pp. 99-112.

Rey Pastor, J. y Puig Adam, P. (1932). Elementos de Aritmética. Col. Elemental intuitiva. Tomo I. Sexta Edición. Madrid: Imp. A. Marzo.

Rey Pastor, J. y Puig Adam, P. (1936). Matemáticas $4^{o}$ curso. Parte $1^{a}$ : Aritmética. $2^{\mathrm{a}}$. Edición corregida. Madrid: Unión Poligráfica, SL. 
Rey Pastor, J., Pi Calleja, P. y A. Trejo, C. (1957). Análisis Matemático. Vol. I. Buenos Aires. Argentina: Kapesluz.

Rico, L.; Lupiáñez, J. L. y Molina, M. (2013). Análisis Didáctico en Educación Matemática. Metodología de investigación, formación de profesores e innovación curricular. Granada: Comares

Schubring, G. (1987). On the Methodology of Analysing Historical Textbooks: Lacroix as Textbook Author. For the Learning of Mathematics. 7, 3, 41-51.

Sesiano, J. (2014). Liber Mahamelet, Heidelberg, New York, Dordrecht, London: Springer.

Sharp, J. (1998). A constructed algorithm for the division of fractions. En Lorna, J. Morrow and Margaret, J. Kenney (Eds.), NCTM 1998 Yearbook: The teaching and learning of algorithms in school mathematics, pp. 204-207. Reston, VA: NCTM.

Sierra, M., González, M. y López, C. (1999). Evolución histórica del concepto de límite funcional en los libros de texto de bachillerato y curso de orientación universitaria (C: O. U): 1940-1995. Enseñanza de las Ciencias, 17(3), pp. 463-476.

Sinicrope, R., Mick, H, W. y Kolb, J.R., (2002). Interpretations of Fraction Division. En Bonnie Litwiller y George Bright (Eds.), NCTM 2002 Yearbook: Making Sense of Fractions, Ratios, and Proportions, pp. 153-161. Reston, VA: NCTM.

Stifel, M. (1544). Arithmetica Integra. Norimberg: Johan Petreium. Bruño.

Valdes, J. y Santos, J. J. (1975). Matemáticas especiales COU. Plan 1975. Madrid:

Valdivia M. y García R. (1969). Matemáticas $3^{\circ}$ curso. Valencia: Bello.

Vallejo, J. M. (1841). Tratado Elemental de Matemáticas. Escrito de orden de S. M. para uso de los caballeros seminaristas del seminario de nobles de Madrid y demás casas de educación del Reino. Cuarta edición corregida y considerablemente aumentada. Tomo I. Parte primera, que contiene la Aritmética y Álgebra. Madrid. Imp Garrayasaza.

Warrington, M. A. (1997). How children think about division with fractions. En Reston, Va. Math. Teaching (Eds.), Mathematics Teaching in the Middle School, Vol. 2. No.6.

Yamaguchi, T. y Jwasaki, H. (1999). Division with fractions is not division but multiplication: On the development from fractions to rational numbers in terms of the Generalization Model designed by Dörfler. En O. Zaslavsky (Ed.), Proceedings of the 23rd Conference of the International Group for the Psychology of Mathematics Education (Vol. 4, pp. 337-344). Haifa: Technion-Israel Institute of Technology.

Agradecimientos. Este trabajo se ha realizado en el marco del proyecto de investigación del Ministerio de Ciencia e Innovación, referencia: EDU2012-35638. Plan Nacional de $\mathrm{I}+\mathrm{D}+\mathrm{i}$.

\section{Referencias de los autores}

Bernardo Gómez Alfonso, Universidad de Valencia (España). Bernardo.gomez@uv.es 
Olimpia Figueras Mourut de Montppellier ${ }^{1}$, Centro de Investigación y de Estudios Avanzados del IPN (México). figuerao@cinvestav.mx

Mauricio Contreras del Rincón (post mortem auctoris), Universidad de Valencia.

${ }^{1}$ Olimpia Figueras ha colaborado en proyectos del grupo del PNA de la Universidad de Valencia desde 1993 en el marco de convenios entre la Universidad de Valencia, España y el Centro de Investigación y de Estudios Avanzados del Instituto Politécnico Nacional, México; en particular codirigió junto con Bernardo Gómez la tesis de Mauricio Contreras. 


\section{Teaching models for algorithms of fraction's división}

Bernardo Gómez Alfonso, Universidad de Valencia (España). Bernardo.gomez@uv.es

Olimpia Figueras Mourut de Montppellier, Centro de Investigación y de Estudios

Avanzados del IPN (México). figuerao@cinvestav.mx

Mauricio Contreras del Rincón (post mortem auctoris), Universidad de Valencia.

Numerical and algebraic thinking group members of Valencia at Spain carry out research projects on the phenomena of teaching and learning of arithmetic and algebraic concepts included in the curricula of primary and secondary education. Among those studies, an investigation on the division of fractions is done. Results related to the teaching models of algorithms of fraction's division are described in this article.

The Local Theoretical Models (LTM) theory is the framework used by the aforementioned group to sustain most of the research projects they are involved in, both theoretically and methodologically. One phase of the construction of a LTM is the description of the existing teaching models in the school system, which is the authors' centre of interest aiming at the characterization of types of algorithms for division of fractions that have starred in mathematics textbooks written from the thirteenth century to the present day.

Constitutive elements of the teaching models for algorithms of fractions' division contained in mathematics textbooks (old and current) are identified focusing on: i) the mathematical contents which play a significant role in the teaching model, ii) the structure of the model, both conceptual and didactical, and iii) the ways used by the mathematics textbook's authors to communicate ideas.

Two types of analysis support the revisiting of mathematics textbooks, the historic-epistemological analysis and the didactic analysis. The latter focused on content analysis considering the conceptual structure, phenomenological aspects and representation systems.

Results obtained from the analysis of mathematics textbooks are organized into two broad categories. One deals with the characterization of algorithms used in the books that were analysed and the other is about the foundations taken into account by the authors to explain the algorithms they employ.

The first category was divided into two classes: i) general algorithms for any two fractions, and ii) particular algorithms that can be used only for pairs of fractions with certain characteristics. Four general algorithms were identified: 1. Reducing fractions to a common denominator and dividing the numerators (Chuquet, 1484 and Perez de Moya, 1562). 2. Multiplying by the inverse fraction or the reciprocal of the divisor fraction (Mahameleth, 1200; Stifel, 1544, and Valdivia and Garcia, 1969). 3. Cross products (José de Zaragoza, 1515). 4. Undoing the fraction by reducing it to a unitary fraction (Dalmau and Carles, 1898).

Two types of algorithms for particular cases were recognized: 1. Cancelling algorithms (Frisus, 1582 and Forcadell, 1585) and 2. Converting fractions to decimals (Hernán and Carrillo, 1988).

Seven types of meanings of the fraction's division were used by the mathematics textbooks authors' to explain the algorithms: i) Division as the number of times the 
divisor equals the dividend was used to explain the algorithm 'undoing the fraction' (Bails, 1818, and Edelvives, 1934). ii) 'Reducing to a common denominator' was explained via the meaning of the division as 'part of' (Rey Pastor and Puig Adam, 1932). iii) 'Reversing the divisor' was based on the construct of Euclidean model (Lacroix, 1825). iv) 'Multiplying by the inverse of the divisor' was explained using the meaning of division as 'reversing multiplication within the missing factor model' (Fernandez and Cardin, 1863; García Roca, 1965, and 2002 Bujanda and Mancilla, 2002); the aforementioned algorithm is also sustained in terms of the solution of an equation (Rey Pastor and Puig Adam, 1932; Rey Pastor, Pi Calleja and A. Trejo, 1957, and Valdes and Santos, 1975). v) 'Cross products' was explained using the meaning of the division as 'the inverse of multiplication' in the multiplicative factor model (Vallejo, 1841). vi) 'Reducing to common denominators' was discussed using 'invariant alterations of a fraction' (Vallejo, 1841, Anzola and Vizmanos, 1997, and Contreras, 2007). vii) The meaning of the division linked to the area model was used to sustain the algorithm 'part of' (Anzola and Vizmanos, 1995 and Ott, Snook and Gibson, 1991).

Overall it is shown that the ideas used by mathematics education researchers to design teaching sequences for the division of fractions that were tested with children are options that were already taken into account by the authors of the mathematics textbooks over time. Consequently, the analysis of mathematics textbooks, old and current, should be considered as a valuable source of inspiration for the design of teaching models for teacher training and curriculum designers. 